\title{
Desafíos del servicio social en el marco de las nuevas políticas sociales
}

\section{Manuel Herrera Gómez}

\author{
Antonio M. Jaime Castillo \\ Universidad de Granada \\ Departamento de Sociología. Facultad de Ciencias Políticas y Sociología \\ mherrera@ugr.es \\ amjaime@ugr.es
}

\section{Resumen}

Tras presentar y analizar las transformaciones más recientes del Estado de bienestar, en este artículo se aborda el sentido de la inclusión social, que puede ser interpretada de diversa forma (según diferentes códigos simbólicos). Posteriormente, se observa que el servicio social debe tener presente el actor de la política social, sus lógicas y las diferentes configuraciones de intervención posibles para realizar su actividad. Y, por último, se diseñan las que podrían considerarse líneas guía y palabras clave de las nuevas políticas sociales.

Palabras clave: Estado de bienestar, políticas sociales, servicios sociales.

\section{Abstract. The Challenge of Social Service in the Social Politics frame}

After presenting and analyzing the most recent transformations in the Welfare State, this article discusses the sense of social inclusion, which can be interpreted in different ways (according to different symbolic codes). Secondly, it is observed that the social service needs to bear in mind the actor of social politics, its logics and the different possible intervention configurations in order to perform its activity. Lastly, the article designs what could be considered as the guidelines and keywords of new social politics.

Key words: Welfare State, social policy, social service.

\section{Sumario}

1. La inclusión social como gran objetivo de la política social

2. Transformaciones estructurales del Estado de bienestar

3. Dos formas de entender la inclusión social: código de la inclusión/exclusión y código de lo relacional/no relacional

4. Tres configuraciones de la política y la intervención social (oferta institucional, mercado regulado y bienestar societario plural) y el principio de subsidiariedad
5. El escenario de las políticas sociales y sus nuevas palabras clave: ciudadanía compleja, subsidiariedad, bienestar asociativo y derechos familiares humanos

6. Bibliografía 


\section{La inclusión social como gran objetivo de la política social}

El siglo XX ha sido una etapa de profundas transformaciones para las sociedades de la Vieja Europa. Los cambios políticos, económicos, sociales y culturales que se han producido durante esta centuria - entre otros, la consolidación de unos modelos democráticos de convivencia, las concentraciones urbanas, el acelerado descenso de la población activa agraria y el incremento en el sector industrial y de servicios, el aumento de trabajo extradoméstico de la mujer, la elevación de las tasas de escolarización de los dos sexos, la secularización, la difusión del bienestar económico y de las sociedades de consumo, el cambio de la estructuras ocupacionales y la reducción del paro, etc. — han permitido el tránsito de unas sociedades industriales a unas dinámicas sociedades postindustriales.

No resulta arriesgado afirmar que, posiblemente, el fenómeno de mayor trascendencia económica, social y política durante esta centuria ha sido el Welfare State (Montoro, 1997). En palabras de Tortella (2000), la verdadera revolución del siglo XX ha sido el desarrollo de esta forma de estado. El Estado de bienestar, con sus diferentes configuraciones ${ }^{1}$, ha articulado la vida social y ha marcado la pauta del progreso económico en los distintos países desarrollados. Esta realidad ha afectado también, y de manera decisiva, al propio Estado, que se ha transformado, con el correspondiente refrendo constitucional en la mayor parte de los casos, en Estado social de derecho.

1. Un interesante y ya clásico estudio sobre la diferente configuración del Estado de bienestar puede encontrarse en Furniss y Tilton (1977). En él se distinguen tres tipos de Estado intervencionista: a) el Positive State, que destaca los seguros sociales, descansa en el individualismo y en la protección de intereses corporativos, no garantiza ninguna forma de sustitución de la propiedad para todos los ciudadanos, excluye de hecho a los obreros que no tengan un trabajo fijo y utiliza la seguridad social como un medio de control social; b) el Social Security State, que persigue una política de pleno empleo, que garantiza a los ciudadanos (incluidos los no asegurados) unos ingresos nacionales mínimos como un derecho y que aspira a una igualdad de oportunidades, no a una igualdad material; c) el Social Welfare State, que descansa en los principios de igualdad, cooperación y solidaridad, que sustituye ampliamente los programas de seguridad social y de ayuda social con servicios sociales públicos, que son iguales para todos, que procura reducir las diferencias en los niveles de sueldos, que aspira al pleno empleo mediante la cooperación entre el gobierno y los sindicatos, y se esfuerza por dar a los trabajadores una influencia política dominante. Como ejemplos de estos tres tipos de estado se citan los Estados Unidos, Reino Unido y Suecia, respectivamente. En nuestra opinión, estos tres tipos de estado quedan demasiado separados unos de otros. En la denominación del Reino Unido como Social Segurity State, por ejemplo, no se toma en consideración que para el sistema británico de seguridad social tiene precisamente una significación básica el National Health Service, un servicio social público accesible en iguales condiciones para todos los ciudadanos, que, sin embargo, es considerado como una característica básica del Social Welfare State. Alemania, en la terminología de los autores, sería clasificada seguramente como un tipo de Positive State, por su acentuación de la seguridad social. Pero también aquí se le garantizan a todos los miembros y a sus familiares amplias prestaciones médicas en iguales condiciones, con independencia de su cuota, por encima del seguro de enfermedad legal que cubre a la inmensa mayoría de la población. Los seguros sociales se juzgan demasiado negativamente, y no se toman en cuenta sus diferencias respecto al principio de los seguros privados. También se define demasiado restrictivamente el concepto de Social Welfare State, es decir, del Welfare State en sentido propio. 
Sin el ánimo de ser exhaustivos, los logros más evidentes del Estado de bienestar han consistido en:

- La implantación de un sistema universal de seguridad y protección social y de otro de garantía de recursos para quienes tienen dificultades de inserción o de mantenimiento en el proceso productivo, y en la provisión, también universal, de un conjunto de servicios y equipamientos colectivos para la salud y para la obtención de unos niveles educativos mínimos.

- La gran estabilidad gracias al papel desempeñado por la intervención estatal en la disolución del conflicto social tradicional (entre patronos y obreros) y en la creciente complejidad de la estructura social, en la que unas clases medias en expansión actúan de colchón entre los extremos de la desigualdad económica. El Estado de bienestar ha conseguido diluir en buena medida la conflictividad social al convertir al Estado en el árbitro de los intereses en disputa, demostrando al mismo tiempo una gran capacidad integradora que ha impedido que la protesta social se convirtiese, como en épocas pasadas, en una rebelión contra el sistema.

$\mathrm{Al}$ asumir estas funciones, dejando de ser un instrumento de los grupos dominantes, el Estado se ha legitimado a sí mismo y ha legitimado al sistema. Según plantea Esping-Andersen (1993), el Estado ha dejado de ser un aparato policial, o un Estado orientado al control, para pasar a ser un Estado productor de servicios, orientado hacia el bienestar de los ciudadanos. Ha resultado ser, en definitiva, el escenario idóneo donde se dirime una buena parte de los conflictos por medio de un poder que está por encima de los diferentes intereses corporativos en benéfico de la armonía social (Herrera, 2001).

Ahora bien, en el tránsito del siglo XX al siglo XXI, Europa, al igual que el resto de sociedades postindustriales, tiene que seguir avanzando y profundizando en los problemas del bienestar, es decir, de la seguridad y la protección social, de los servicios sociales y sanitarios, de la lucha contra las antiguas y nuevas formas de pobreza, mediante políticas de creciente «inclusión social». Dicho en otros términos, se abre una nueva fase histórica en la que es preciso reflexionar sobre el sentido de la protección social y cómo realizarla en un nuevo escenario cuyos rasgos distintivos son:

- Una creciente desestructuración (disgregación) del tejido social: los individuos se encuentran ubicados en escenarios mucho más complejos; el principal reto que debe afrontar Europa es dar una respuesta a dicha complejidad, es decir, las sociedades postindustriales deben ofrecer respuestas a: los desequilibrios demográficos (Iglesias de Ussel, 1998); los cambios derivados del desarrollo económico y las consiguientes modificaciones en la estratificación social (Renaut, 1989); las nuevas patologías de la modernidad (aumentan los estados de malestar psicofísicos, las toxicomanías, las nuevas epidemias como el sida); el individualismo y el privativismo, y las nuevas migraciones (Ardigo, 1980). 
- Una saturación del modelo de Estado social como sistema basado en el compromiso neocorporativo, por tanto, en sistemas de seguridad centralizados y con base fiscal. Decir «saturación» no significa, obviamente, decir que los sistemas centralizados de bienestar por vía fiscal no sean útiles, tan sólo que su radio de actuación encuentra dificultades crecientes. Emergen nuevos problemas a los que el Estado difícilmente puede hacer frente, ya que no están relacionados con el mercado de trabajo ni la producción, como la soledad de los ancianos, las nuevas toxicodependencias o las situaciones de riesgo derivadas de la desestructuración familiar (la pobreza en las familias monoparentales, la violencia doméstica, etc.).

- La crisis del Estado nación. Ésta comporta, entre otras cosas, por una parte, tendencias hacia el localismo y el regionalismo, por otra, creciente internacionalización de los problemas sociales. Ejemplo de esto son algunos fenómenos que tienen repercusiones globales como las crisis económicas, el problema de la seguridad o el deterioro medioambiental.

- Por último, emerge la solicitud de humanización de los ámbitos de vida y de las organizaciones de trabajo y servicio, con la demanda de nuevos derechos sociales ligados a los estilos de vida cotidiana y, en concreto, a la asistencia de las personas.

En este nuevo escenario, la inclusión (o bien cohesión) social es la palabra clave de las políticas sociales europeas (especialmente tras la Declaración de Lisboa). Su significado y la articulación de los instrumentos para su consecución son un problema en el que se asienta el núcleo de los desafíos que se plantearán al servicio social en los próximos años (Wilensky, 1985; Alder, 1998).

Europa presenta una serie de rasgos distintivos que merece la pena señalar. Entre otros: la diferente polity institucional del bienestar, la estructura del gasto social, las diferencias entre los distintos países, etc. Sin embargo, una vez realizadas las comparaciones apropiadas con el resto de sociedades avanzadas, una de las diferencias más interesantes reside en la dificultad de los sistemas de bienestar de la Vieja Europa para producir procesos de diferenciación y de articulación relacional en el complejo campo de las políticas sociales, y en concreto de los servicios de importante contenido relacional para:

- Equilibrar la individualización de las intervenciones con la producción de nuevas solidaridades, ya sea entre los destinatarios de tales intervenciones, ya sea entre los sujetos implicados en la ayuda social ${ }^{2}$.

- Mantener conexiones significativas entre todas las necesidades de la vida cotidiana a la luz de un concepto de bienestar "como vida buena», que requiere, en concreto, la movilización de los recursos potenciales y laten-

2. La primera reorientación es la siguiente: las nuevas políticas sociales deben promover los derechos de la persona mediante esferas sociales intermedias que están en contacto directo con los mundos de la vida cotidiana. Se trata de producir una nueva combinación entre universalismo y pertenencia. A este respecto, H. S. Falck (1988) habla de una nueva concepción del trabajo social como gestión de las pertenencias (membership). 
tes de los sujetos concebidos no como individuos aislados, sino como elementos de redes más complejas de apoyo en continua actividad de asistencia ${ }^{3}$.

- Tener como referente operativo no al individuo, sino a la familia como sujeto social en el que las necesidades encuentran su sentido cualitativamente más significativo y la primera respuesta ${ }^{4}$.

- Coordinar las diferentes intervenciones de bienestar y asistencia, de tal forma que la combinación de los diversos factores compense y resuelva las eventuales carencias de una particular dimensión o aspecto del problema a afrontar ${ }^{5}$.

Tras presentar las transformaciones del Estado de bienestar, en estas páginas abordaremos el sentido de la inclusión social, que puede ser interpretada de diversa forma (según diferentes códigos simbólicos); posteriormente, observamos que el servicio social debe tener presente el actor de la política social, sus lógicas y las diferentes configuraciones de intervención posibles para realizar su actividad, y, por último, diseñamos las que consideramos líneas guía y palabras clave de las nuevas políticas sociales.

\section{Transformaciones estructurales del Estado de bienestar}

Los orígenes de los sistemas de bienestar se sitúan en un contexto histórico específico, el de la postguerra mundial europea. Las transformaciones económicas, demográficas y sociales que se producen en las sociedades industriales avanzadas en la segunda mitad del siglo XX tienen efectos de gran calado sobre la configuración del Estado de bienestar y la definición y articulación de los mecanismos de protección social.

De una parte, los niveles de demanda dirigidos al Estado de bienestar crecen a un ritmo exponencial. Siguiendo la lógica de Daniel Bell (1994), el

3. En el nivel de las intervenciones macroinstitucionales, se habla de intersectorialidad. Con este término, se entiende que, cuando se decide perseguir un objetivo de política social, se debe evaluar tal objetivo desde el punto de vista de todos los sectores implicados. En el nivel de las intervenciones microsociales, se habla de trabajo de redes. El bienestar es mayor cuanto más significativas y operativas sean cultural y funcionalmente las redes de apoyo de las personas (Karpel, 1986).

4. Son muchas las investigaciones empíricas que han demostrado que los individuos combinan recursos y estrategias, contratan los servicios, negocian las formas de bienestar con los operadores formales, en función de estrategias que no son atomistas (individuales), sino del grupo familiar de pertenencia (Bulmer, 1987). Todo ello demuestra que es en el nivel de la estructura familiar donde el bienestar tiene su más significativa integración cultural.

5. La teoría de los mecanismos de acuerdo (linkage mechanisms) entre intervenciones microsociales de tipo informal e intervenciones institucionales formales para satisfacer un radio más amplio de necesidades sociales es, en este sentido, crucial (Litwak, 1985). Puede ser generalizada, con las oportunas adecuaciones, desde el campo de ayuda a los ancianos al resto de sujetos socialmente débiles (discapacitados, enfermos, infancia en situaciones de riesgo, etc.). 
Estado se ha convertido en núcleo de imputación de todas las demandas privadas en un proceso de "publificación" de los problemas privados, por el que los grupos de interés compiten en el mercado político por los recursos del Estado para resolver sus problemas.

En la medida en que, durante las fases iniciales del Estado de bienestar, éste admitió la legitimidad de las demandas de realización de derechos, difícilmente podría controlar luego la espiral subsiguiente. Las demandas no son sólo ya de los grupos menesterosos de la sociedad, sino de todo tipo de grupos que requieren la acción del Estado en defensa de intereses (Bell, 1994).

A pesar de la evolución explosiva de las demandas, los recursos del Estado no pueden evolucionar en la misma dirección. El rápido crecimiento de la presión fiscal en las economías de la OCDE en las décadas posteriores a la Segunda Guerra Mundial se hizo insostenible con las crisis económicas de la década de los años setenta. Se produjeron movimientos de protesta fiscal en muchos lugares, debido a la alta presión fiscal que soportaban las clases medias.

Las crisis económicas también mostraron la imposibilidad de avanzar por una senda de déficits públicos expansivos que tenían efectos potencialmente negativos para el mantenimiento de la prosperidad económica en un entorno más adverso. La disciplina fiscal y presupuestaria se introdujo como una máxima de la política económica. En definitiva, nos movemos, a partir de entonces, en un terreno donde los recursos económicos del Estado se ven limitados, y esta situación no parece previsible que cambie en el corto y medio plazo.

Estos fenómenos plantean la necesidad de una readaptación de las estructuras de bienestar. En primer lugar, en sentido amplio, se observa una tendencia a la racionalización del gasto en políticas sociales. No se trata tanto de recortes del gasto como de mejorar la eficacia y la eficiencia de las partidas presupuestarias destinadas a la promoción social. La racionalización del gasto social se ha llevado a cabo mediante tres mecanismos fundamentales:

- En primer lugar, el ahorro de los costes de gestión de los servicios. Este proceso ha llevado a una separación entre la titularidad pública de las prestaciones del Estado de bienestar y los procesos de gestión. El Estado ha mantenido la titularidad de las prestaciones, lo cual implica la garantía y la responsabilidad estatal de los servicios. Pero, al mismo tiempo, se han introducido mecanismos de gestión de los servicios públicos en los que tienen cabida otros actores sociales, distintos del Estado, como las empresas privadas o las organizaciones voluntarias. Esto supone una reducción de los costes de gestión, a través de la externalización, sin que por ello se produzca una merma de la calidad asistencial. La creciente presencia de las organizaciones voluntarias en la gestión de los servicios hace posible una mejor atención a las demandas concretas de grupos específicos de población, las cuales no podían ser efectivamente resueltas dentro del marco universalista de estandarización de los servicios del Estado de bienestar (por ejemplo, minorías étnicas o religiosas). 
- En segundo lugar, hay un proceso de descentralización de la gestión de los servicios hacia los niveles más cercanos de la Administración pública. En la generalidad de los países occidentales, aún con las diferencias que imponen los diversos sistemas político-administrativos, más o menos descentralizados, las competencias en la prestación y gestión de los servicios de bienestar se han trasladado hacia las administraciones de nivel inferior, las de ámbito local o regional. Buen ejemplo de ello es el caso español, en donde las competencias de planificación sobre los servicios sociales están transferidas a las comunidades autónomas, según establece la norma constitucional. Además de ello, la legislación sobre régimen local atribuye a las entidades locales, ayuntamientos y diputaciones provinciales, un buen número de competencias de ejecución en materia de servicios sociales. El objetivo de este proceso de descentralización es llevar la gestión de los servicios de bienestar a los niveles de decisión más cercanos al ciudadano, y por lo tanto más cercanos a la realidad social y económica sobre la que operan estos servicios, con lo que mejora la eficiencia en la asignación de los recursos y la calidad de las prestaciones.

- A un nivel más general, está cambiando la propia concepción acerca del papel de las políticas sociales. Desde una lógica puramente asistencial, las políticas sociales evolucionan hacia una suerte de corresponsabilidad. Los programas de bienestar están orientados hacia una inserción social plena de los beneficiarios, antes que hacia una política de paliación de situaciones de precariedad social por vía exclusiva de instrumentos de sustitución de rentas. Hay dos fenómenos estrechamente conectados con este proceso. Por una parte, en la opinión pública parece percibirse de manera cada vez más clara que la justificación de los programas de bienestar está en su efectividad práctica y no en contenidos ideológicos abstractos. Así, señala Tom W. Smith (1995), los ciudadanos no pretenden recortar el apoyo público hacia las personas en situación de necesidad, sino que favorecen tanto gasto como sea necesario para atender a las necesidades sociales, pero igualmente cuestionan las medidas concretas de política social que ponen en marcha los gobiernos. Los criterios de evaluación del gasto público por parte de la ciudadanía se han vuelto más pragmáticos. La sociedad ha tomado conciencia de la necesidad de hacer un empleo eficaz y eficiente de los recursos públicos. Por otra parte, se habla cada vez más de «sociedad del bienestar», en lugar de "Estado de bienestar». Este cambio semántico hace referencia a la necesidad de otorgar un mayor protagonismo a los servicios sociales producidos no sólo por el Estado, sino también por otros actores sociales, fundamentalmente las organizaciones voluntarias y las familias. En las sociedades contemporáneas complejas, la producción de servicios de bienestar no es una tarea exclusiva de las administraciones públicas, sino que implica al conjunto de la colectividad social. La aportación que pueden realizar las organizaciones del llamado "tercer sector» y las propias familias es capital. En este sentido, las medidas de política económica han de dirigirse a esti- 
mular a la sociedad civil y al tejido asociativo, a través de medidas de carácter fiscal y de promoción y colaboración con las entidades sin ánimo de lucro. Por otro lado, el apoyo a las familias, como agentes productores de servicios de bienestar, es capital, dado que sus contribuciones al bienestar conjunto de la sociedad, si bien no son evaluables económicamente por procedimientos directos, tienen una enorme trascendencia desde el punto de vista social y económico.

Estas transformaciones nos sitúan ante un nuevo escenario de los sistemas de bienestar, en el que cambian los códigos simbólicos de la relación del intercambio que se define entre los individuos y los servicios de bienestar, y que se articula a través de las medidas concretas de política social. La necesaria reestructuración de las políticas sociales para adaptarse a unas nuevas sociedades en rápidos procesos de transformación hacen necesario cambiar tanto los objetivos de las políticas sociales como el punto de vista desde el que se analizan y se evalúan sus rendimientos.

\section{Dos formas de entender la inclusión social:}

\section{código de la inclusión/exclusión y código de lo relacional/no relacional}

Existen muchas formas de entender la inclusión social. Incluso dentro de un mismo código de significados, existen diferentes formas de hacerla operativa, implementarla y evaluarla. Sírvanos de ejemplo el siguiente elenco de semánticas de la inclusión social (Di Nicola, 1998):

a) Inclusión como pertenencia. En el sentido más general del término, estar incluido significa "estar insertado como miembro en una comunidad», incluso como comunidad de discurso o como sistema comunicativo.

b) Inclusión como reconocimiento. La inclusión como pertenencia implica determinados criterios de reconocimiento. Se pertenece a una cierta sociedad o comunidad a partir de títulos o cualidades que deben ser reconocidas (por ejemplo, se pertenece a la sociedad de las familias si se está casado o si la forma de convivencia es definida como familia). Las pertenencias son objeto de procesos de reconocimiento que trasladan a específicos códigos simbólicos.

c) Inclusión como criterio de selección. Inclusión significa "posibilidad de acceso a un sistema social cuando se tiene una característica de especial relevancia para el reconocimiento» (por ejemplo, la lengua es un factor de inclusión en una reagrupación social y para determinados requisitos de ser o llegar a ser ciudadano de un estado).

d) En términos generales, podemos decir que toda indicación implica una inclusión o exclusión: el hecho de indicar algo como propiedad o cualidad significa trazar una distinción con quien no la tiene (y por tanto está excluido). 
Se nos puede preguntar: ¿cuál es el código semántico de la inclusión en las políticas sociales? La modernidad ha sido tajante: desde el punto de vista de las políticas sociales, la inclusión social es la plena pertenencia al Estado de bienestar nacional, es decir, gozar del pleno acceso al conjunto de derechos y deberes de la ciudadanía social ${ }^{6}$. Este tipo de inclusión puede, según Bulmer, obtenerse por diferentes vías: a) mediante titulaciones de beneficios (entitlements) otorgadas a los portadores de derechos de ciudadanía, o b) mediante apoyos para que todos puedan participar en la competición por los recursos sociales a partir de una estructura generalizada de igualdad de oportunidades (Bulmer, 1989). Por tanto, inclusión social significa la posibilidad de concurrir para obtener los mejores beneficios por el hecho de pertenecer a una determinada sociedad política, gozando de sus formas de protección social.

Éste es el código moderno de la inclusión. Actualmente, dicho código experimenta una importante transformación debido a una serie de factores. En concreto, por la dificultad de llevar a cabo la inclusión mediante la extensión de titulaciones y activar eficientemente una estructura igualitaria de oportunidades en la participación competitiva (Midgley, 1997). Estas dificultades pueden ser reconducidas a dos grandes procesos: la reversibilidad de los criterios y el mayor atravesamiento (crossing) de los límites planteados en las distinciones que definen los dos campos, el de los incluidos y el de los excluidos. Quien estaba incluido puede llegar a convertirse en un excluido. Muchos incluidos en un sector de beneficios son excluidos de otros. El Estado actúa como «una institución que intenta corregir la arbitrariedad». Un criterio de pertenencia puede decaer y emerger uno diferente. Se puede estar incluido en

6. No está de más recordar la clásica tesis de Marshall (1950), que procede al análisis de la emergencia de la ciudadanía en el marco del Estado nación moderno en términos de desarrollo de la sociedad capitalista. Por ciudadanía entiende un estatus vinculado a la plena pertenencia a la comunidad política. De aquí que aquéllos que posean dicho estatus sean iguales en relación con los derechos y los deberes asociados a la propia existencia de la comunidad. Su aportación más significativa es la conciencia de trascender la idea de que la pertenencia a una comunidad política es una cuestión de carácter político. Es por ello por lo que Marshall introduce la que quizá sea su aportación más conocida: la clasificación de los tres elementos de la ciudadanía. El primero, el civil, se compone de los derechos indispensables para el ejercicio de las libertades individuales, de palabra, de pensamiento y de creencia, el derecho a poseer las cosas en propiedad o de estimular contratos válidos, además del derecho a una justicia imparcial. La institución directamente asociada con ellos es el sistema de tribunales de justicia. El reconocimiento de este cuerpo de derechos es fundamental para el desarrollo de la economía libre de mercado. El elemento político comprende el derecho a participar en el ejercicio del poder político bajo las formas y los modos permitidos. Por consiguiente, está vinculado de forma directa con la aparición de instituciones parlamentarias. Su resultado más visible es la ampliación paulatina del sufragio hasta alcanzar el denominado «sufragio universal». Por último, el social agrupa toda una gama que va desde un mínimo de bienestar y seguridad económica hasta el derecho a participar plenamente en la convivencia social y a vivir la vida de personas civiles según los cánones vigentes en la sociedad. Estos derechos se realizan principalmente por medio de los servicios sociales y el sistema educativo. Un interesante y reciente estudio sobre la tesis de Marshall puede encontrarse en Morán y Benedicto (2000). 
función de diversos aspectos, pero no por otros. La complejidad es aún mayor por el hecho de que cada modalidad de inclusión comporta una pluralidad de formas de exclusión, a las que no se puede poner remedio a partir del principio que las ha generado. Por ejemplo, incluir mediante el instrumento de un ingreso mínimo garantizado puede conducir a excluir al beneficiario del trabajo o de adoptar ciertas iniciativas o utilizar determinadas oportunidades, exclusiones que no se pueden remediar a través de posteriores ganancias de ingresos. $\mathrm{O}$, viceversa, incluir en la sociedad mediante un trabajo garantizado puede conducir a excluir al beneficiario de ciertas acciones que podrían aumentar sus ingresos o permitirle la adopción de ciertas actividades o servicios.

Todo ello produce la transformación del código moderno de la inclusión social y genera un nuevo código —el postmoderno-, que está caracterizado por la posibilidad de que los criterios de inclusión y exclusión se hagan reversibles o que lleguen a transformar los incluidos en excluidos.

Pero no sólo esto. En la condición postmoderna surge otra situación: se verifica que las distinciones de pertenencia pueden cambiar radicalmente sus criterios. Si cambian los criterios a partir de los que se está incluido o excluido, el código de la inclusión no es ya el moderno o el postmoderno, sino que se hace transmoderno (Donati, 2002). Esto se verifica, por ejemplo, cuando los criterios de inclusión aluden a una pertenencia que ya no es la de la ciudadanía estatal, sino la de la pertenencia a reagrupamientos sociales autoorganizados. La inclusión tiene lugar no bajo la protección del complejo de la ciudadanía moderna, sino bajo la protección más o menos elegida de contextos policéntricos de tipo asociativo.

Por tanto, tenemos que trabajar con nuevos códigos de inclusión y exclusión, diferentes de los modernos y postmodernos, que podemos denominar «transmodernos». En buena medida se trata de códigos más latentes que manifiestos, que modifican el sentido del espacio público y redefinen las modalidades de inclusión social, en términos que no se corresponden ya a lo estatal moderno, sino que son policéntricos (como diría G. Teubner, 1992), es decir, que reflejan códigos diferentes para todo contexto.

Del código inclusión y exclusión se pasa al código relacional y no relacional: es decir, se está incluido en una política social o familiar no por el hecho de pertenecer a un estado, sino por estar insertado en formas de pertenencia más generales que valoran contextos relacionales incluso no estatales. La característica fundamental del nuevo código simbólico que orienta las políticas familiares consiste en que la inclusión no tiene lugar como forma de protección por parte del Estado social, sino como forma de conexión de un individuo según un código multidimensional que diferencia las pertenencias y los derechos y deberes, o bien los costes-beneficios, de las singulares pertenencias, incluidas las familiares. La conexión no es ya dicotómica (se está incluido o excluido), sino que se declina relacionalmente, es decir, selectivamente: lo que significa que un sujeto sólo puede ser incluido en determinados aspectos y no en otros, bajo determinadas condiciones, en una situación y por un cierto tiempo. 
A ello le corresponde un cambio en el código de la «normalización». En la modernidad, el código de la normalización es de tipo estatal, es decir, tiene lugar como extensión de un cierto intervalo de la distribución estadística gaussiana de las situaciones y de los comportamientos admitidos y titulares de reconocimientos y beneficios. Se constata que el proceso capitalista, sin las oportunas correcciones, produce pobreza, desigualdad, marginación, y se plantea el problema del límite de aceptación de estas situaciones. La tendencia a incluir tiene lugar como proceso de normalización de estas situaciones mediante la extensión de ciertas provisiones que permiten la entrada en la norma de la distribución de los recursos ${ }^{7}$. Sólo quien no quiere o puede hacerse incluir queda marginado (lo que equivale a decir que no se "normaliza») (Sgritta, 1990).

En la transmodernidad, la normalidad no puede entenderse de esta forma. Decae la idea de que las situaciones y los comportamientos sociales puedan ser confrontados con una distribución estadística. En una situación de creciente fragmentación, individualización, complejidad, la normalidad debe ser redefinida: se plantea el problema cultural del reconocimiento de la especificidad de una situación y de un trato relacional adecuado (es decir, adecuado a la definición relacional de aquella situación o de aquel comportamiento). La normalidad se convierte en un problema de adecuación relacional al «caso singular» (Payne, 1995). Las políticas familiares constituyen un campo emblemático de este cambio.

Con el nacimiento del nuevo siglo, las políticas sociales están marcadas por un dilema fundamental: escoger entre políticas sociales que asumen el criterio de una inclusión basada en la neutralidad ética (están en función de la neutralidad ética), y políticas sociales que asumen un criterio de inclusión basado en elecciones éticas (están en función de una conexión ética entre los ciudadanos a especificar), que podríamos denominar «solución relacional» (Banks, 1995) (tabla 1).

El código relacional nace y se desarrolla para suplir a los fracasos del código de la inclusión estatal, pero ésta no es su función o característica principal. Traslada a otra forma de concebir las políticas sociales. El lector lo podrá constatar comparando los ocho indicadores de la tabla 1.

El código relacional tiene como principio observar si la medida de inclusión planteada en un sistema social no genera exclusiones. Y lo verifica contemplando los efectos en términos de relaciones, ya que realizar un derecho individual no implica que beneficie a las relaciones familiares o sociales que posee una persona (Sanicola, 1997). Por ejemplo, las asignaciones por maternidad

7. Como bien ha señalado Ardigo, la normalización llevada a cabo por la modernidad significa introducir correcciones no marginales a la acción del mercado, pero manteniendo al mismo tiempo inalterables los requisitos del sistema económico capitalista. Dentro de este compromiso, la normalización puede ser leída como una corrección eficaz. Más concretamente, supone una alteración de la estructura de la desigualdad social y hace más difícil la permanencia de las situaciones de exclusión producidas por la economía de mercado (Ardigo, 1997). 


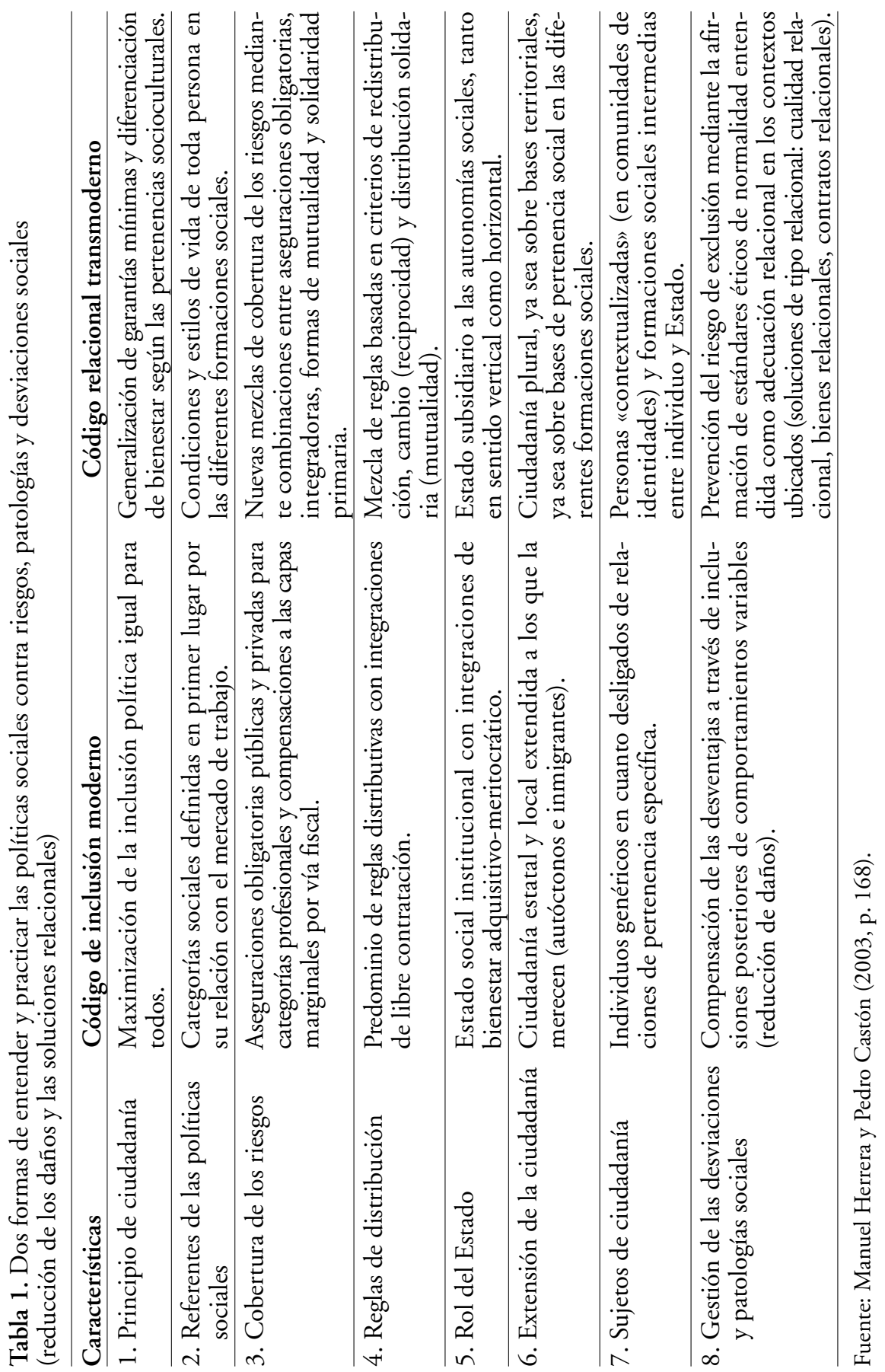


pueden otorgarse con el objetivo de incluir socialmente a la mujer en un sistema de protección social. El código de inclusión se considera satisfactorio si se aprueba una ley en este sentido. El código relacional pide más: observa si esta medida efectivamente crea relaciones de solidaridad o si, por el contrario, tiene el efecto de aislar a la mujer. En el segundo caso, replantea tal medida según la posibilidad de utilizarlo de diversa forma: con servicios (en king) y no sólo en dinero (en cash), y a través de una configuración asociativa y no mediante anónimas contrataciones, es decir, contextualizándolo en una red de comunidad.

El código relacional tiene como objetivo realizar lo que se ha llamado «ciudadanía profunda» (deep citizenship: Clarke, 1996), es decir, una ciudadanía concreta y contextualizada en una comunidad peculiar.

$\mathrm{Al}$ mismo tiempo, trata de remediar los límites y las carencias del código de inclusión, en el que muchos welfare benefits incluyen a los individuos en un determinado sistema de garantías (por ejemplo, ingresos mínimos), pero los aíslan desde el punto de vista de las relaciones (familiares o sociales). Mientras que los códigos no relacionales se preguntan «quién» deben incluir (según reconocimientos, selecciones e indicaciones de pertenencia estatal), y consiguientemente excluyen a los que no poseen determinados requisitos; los códigos relacionales se preguntan "cómo» incluir toda persona y familia, haciendo que el camino de la pertenencia relacional represente una más amplia y mejor modalidad de actuación de los derechos humanos fundamentales. Por tanto, mientras que los códigos no relacionales conllevan el hacer funcionalmente equivalentes las funciones familiares y los núcleos de solidaridad, los códigos relacionales analizan tales funciones y núcleos a partir de la calidad de las relaciones que presentan.

\section{Tres configuraciones de la política y la intervención social (oferta institucional, mercado regulado y bienestar societario plural) y el principio de subsidiariedad}

Los actores de las políticas sociales son de tres tipos: estatales, corporativos y a redes. Cada uno tiene su propia lógica: respectivamente, de mando jerárquico (normas condicionales y preceptivas, basadas en el poder y la ley), de intercambio social de mercado (profit y non profit) y de reciprocidad reticular (Barry, 1990). El trabajador social debería comprender que trabajar para alguno de estos actores (y sus códigos simbólicos) comporta enormes diferencias de formación, acción, implementación, verificación y supervisión.

A causa de su diferente naturaleza, los «entes» que hacen política e intervención social pueden adoptar no una, sino una pluralidad de configuraciones de política social. Les llamaremos, por brevedad, de esta forma:

- Configuraciones de oferta institucional.

- Configuraciones de mercado regulado.

- Configuraciones de bienestar societario plural. 
Conviene matizar que dejamos a un lado aquella configuración que la literatura especializada llama de "altruismo compasivo», en cuanto que representa una forma de política social y familiar residual, que confía la intervención a organizaciones filantrópicas, de puro altruismo o beneficencia.

Veamos estas tres configuraciones de forma más analítica. En cada una de ellas, el servicio social debe ser capaz de captar las profundas, y en ocasiones radicales, diferencias de los perfiles profesionales y de los estilos de intervención.

El criterio que sugerimos para distinguir estas configuraciones es la forma en que conciben la subsidiariedad ${ }^{8}$, principio fundamental de política social que activa una redistribución vertical, horizontal o mixta. Dicho en otros términos, las tres configuraciones son modalidades diferentes de intervención subsidiaria: respectivamente, vertical (oferta institucional), horizontal (mercado social regulado) y mixto (bienestar societario plural).

\section{A. Oferta institucional}

Aquí, el argumento de política social se inicia con la siguiente pregunta: ¿Qué debe dar el Estado social a los ciudadanos y cómo debe ofrecer mejores servicios? Los objetivos de la oferta institucional esencialmente son garantizar un paquete de bienestar mínimo, de vida decente, a toda persona y a toda familia mediante criterios de redistribución vertical (establecimiento de impuestos desde un centro que actúa - mediante el sistema fiscal general - para compensar las desventajas, redistribuyendo recursos y ofreciendo ayudas sociales a los menos garantizados).

Dos ejemplos aclararán este tipo de configuración, concretamente en el campo de las políticas familiares: 1) el caso de los llamados «paquetes familia», ofertados y garantizados por las administraciones locales; 2) las políticas redistributivas (garantías de ingreso mínimo familiar) por medio de índices de equidad e intervenciones asistenciales (ingreso mínimo de inserción).

La oferta es llamada «institucional» por corresponder al Estado, central, autonómico o local, la responsabilidad en tal sentido, aunque puede llevar a cabo colaboraciones con otras instituciones sociales.

\section{B. Mercado social regulado}

Aquí, el argumento de política social se inicia cuando se plantea este otro interrogante: ¿Es el Estado el mejor gestor de la redistribución social a los ciudadanos y a las familias? ¿Por qué el Estado debe gestionar todo el sistema de bienestar? Y se responde diciendo que el Estado, al menos en ciertas funciones, no es el mejor juez y distribuidor del bienestar, sino que debe limitarse a desarrollar funciones de canalización, control y, puntualmente, financiación, dejando que otros sujetos organicen y gestionen los servicios y las prestaciones de bienestar dentro de un mercado libre de demanda y oferta. La confi-

8. Sobre el principio de subsidiariedad, véanse Cottini (1997) y Rossi (1997). 
guración del mercado social regulado privilegia la redistribución horizontal y se basa en algunas reglas fundamentales (Donati, 2002). Ahora bien, aunque privilegie la redistribución horizontal, sin embargo, cuando es utilizado por instituciones estatales para favorecer a los más débiles, sigue estando presente la redistribución vertical (garantizada por la oferta institucional).

Ejemplos de esta configuración son los denominados de welfare mix, entendidos como compromiso entre regulación institucional y oferta privada; o incluso los llamados «bonos» (vouchers) o títulos de acceso del cliente a un mercado de empresas acreditadas o certificadas, incluso para servicios de pago vinculados mediante convenios con entes públicos (De Leonardis, 1996).

Este modelo produce una progresiva reducción de las distinciones entre profit y non profit (es decir, una cierta homologación de las dos formas organizativas de intervención social). Esto se debe a que la regulación del mercado activada por el Estado privilegia la forma de empresa económica como forma organizativo-jurídico-económica convencionada y ubica a los sujetos del tercer sector (non profit) bajo categorías contractuales más o menos análogas, salvo el hecho de conferir algunas desgravaciones fiscales. En otros términos, la regulación del mercado por parte del Estado comporta que las políticas sociales presten atención a:

1. Más a los output (la singular prestación material) que a los outcome (el desarrollo dinámico de la respuesta a la necesidad).

2. Una acentuada racionalización de los servicios, con énfasis en la profesionalización.

3. Una presión hacia la estandarización de los servicios.

De esa forma, las políticas sociales que asumen la configuración del mercado social regulado pueden incurrir en una creciente mercantilización de los servicios, ya que éstos son intercambiables a partir de un precio monetario (Colozzi, 1987).

\section{Bienestar societario plural}

El argumento de política social de bienestar societario plural comienza cuando nos preguntamos: ¿ Se pueden organizar intervenciones de política social que no sean impuestas por leyes (o requieran la imposición del poder político), ni que respondan a lógicas de mercado (aunque regulado), sino promovidas por formas autoorganizativas (civiles) de solidaridad social?

El bienestar societario plural es la respuesta positiva a esta pregunta. Esta configuración, en otros términos, exalta la función política del empresario social que crea una organización de servicios que produce bienes relacionales ${ }^{9}$, como

9. Se trata de un tipo de bienes que sólo pueden ser producidos y disfrutados conjuntamente por los que son al mismo tiempo productores y usuarios, por ejemplo, la solidaridad y el intercambio simbólico. En buena medida, presentan cierta semejanza con los bienes relacionales primarios, que tienen su significado fundamental en las redes de solidaridad 
son, por ejemplo, una escuela o un servicio de asistencia domiciliaria o preescolar. Al hacer esto, se revela que, aunque los «entes públicos» gozan de la presunción de representar a los intereses generales, en alguna ocasión activan discriminaciones; y aunque gozan del apoyo de los poderes públicos, a veces consumen más recursos de cuanto producen. Se reivindica que otros sujetos sociales (formaciones sociales intermedias como las organizaciones de privado social) pueden conciliar mejor los intereses generales con los particulares, tienen acceso a los propios recursos privados, responden mejor a las exigencias intersubjetivas de los servicios relacionales. Su problema está en que, faltando una presunción de "publicidad», no tienen un acceso fácil a autorizaciones, financiación, créditos y todo tipo de recursos o ventajas disponibles en el patrimonio de una comunidad que, sin embargo, son dados con carácter automático a los entes estatales.

La configuración societaria actúa para la promoción de una vía alternativa al Estado y al mercado. Hasta ahora, al privado social se le han reconocido dos roles: a) el de integrador del ente público, es decir, como intervención en aquellas áreas y problemas en las que lo público no consigue llegar; b) el de un sujeto funcionalmente diferenciado por áreas de intervención respecto a otros actores (en concreto, los servicios públicos), es decir, dividiendo los campos de intervención con los servicios organizados por el Estado, la comunidad autónoma y los entes locales.

La configuración del bienestar societario plural da un paso hacia delante: entiende el privado social como ejercicio de un derecho y un deber de solidaridad, y como solución «estatuaria» que debe ser ubicada en el mismo nivel que los servicios de la Administración pública ${ }^{10}$.

Optar por una solución estatuaria para las esferas de solidaridad social (tercer sector, privado social), que las ubique en situación de autonomía y reciprocidad entre sí y con el ente público significa apostar por estrategias sinérgicas en las intervenciones de política social dirigidas hacia las personas en el contexto de sus familias.

Esta concepción traslada a una concepción de las políticas de servicios sociales, tanto generales como personales, que hace de bisagra con una red inte-

primaria (familia, redes de parentesco y amistad). Este tipo de bienes es totalmente diferente a los producidos por el Estado y los producidos por el mercado. El primero produce bienes públicos que, en principio, no son relacionales en cuanto que implican formas de gobierno y agregaciones de carácter impersonal, formal y abstracto. El segundo produce bienes privados, que no son relacionales por definición porque no implican necesariamente compartir.

10. Por solución estatutaria se entiende una nueva regulación que permita engarzar las organizaciones de la sociedad civil con las grandes estructuras — por más que aligeradas, descentralizadas y flexibilizadas - del Estado de bienestar, a fin de que puedan estar en condiciones de conseguir los medios imprescindibles necesarios para la consecución de sus objetivos. Las regulaciones habrán de ser autónomas, pero amparadas y protegidas por la Administración pública, cuya misión no es sustituir a la sociedad civil y a sus organizaciones, sino ordenar los marcos generales de la convivencia, según la función de coordinación y configuración que le compete (Llano, 1999). 
grada de asistencia de comunidad (community care) ${ }^{11}$. Nace y se desarrolla cuando se es consciente de que la ideología de la "participación política» es una etiqueta para tantos usos incorrectos y conduce a un diagnóstico equivocado de la forma de operar de los sistemas sociales. Los excluidos necesariamente pueden no ser víctimas si tienen la posibilidad de organizar los propios sistemas sociales. Por tanto, esta configuración se basa en otros principios (Donati, 2002). Su función consiste —en términos de subsidiariedad-en realizar una redistribución mixta (conjuntamente vertical y horizontal) en el interior del propio radio de acción.

Ejemplos en este sentido son las mutual organization y las public organization creadas por las familias, como los sistemas organizados por una pluralidad de sujetos autorregulados (por ejemplo, una fundación más un banco más un conjunto de cooperativas de solidaridad social).

\section{El escenario de las políticas sociales y sus nuevas palabras clave: ciudadanía compleja, subsidiariedad, bienestar asociativo y derechos familiares humanos}

En las sociedades complejas de la Europa contemporánea, el Estado social no puede ni debe ser desmantelado, sino que debe ser repensado, tanto en sus finalidades, como en sus instrumentos. Éste es precisamente el objetivo hodierno de las políticas sociales, que requieren nuevas iniciativas y reorganizaciones "desde abajo» ${ }^{12}$.

Las transformaciones que está experimentado el Estado social deberían ir en la línea de captar y valorar toda una serie de movimientos de la sociedad, expresados mediante nuevas formas de solidaridad y de ayuda para finalizar en la construcción de una «sociedad del bienestar» (Ardigo, 1990). Desde ahora,

11. Resulta verdaderamente imposible datar el nacimiento de la expresión community care. Utilizada a finales de los años cincuenta para denominar, en el ámbito anglosajón, las nuevas directrices en el campo de las enfermedades mentales, en principio la expresión indicaba la expansión en el territorio de los servicios sociales del ente público. Con posterioridad, a finales de la década de los setenta, la expresión también viene sistemáticamente utilizada en el ámbito de los informes oficiales sobre los servicios sociosanitarios. Como bien señala Bulmer (1998), aunque en la legislación específica no es posible encontrar una definición del término community care, en él se agrupan una amplia variedad de intervenciones y formas asistenciales. En concreto, la expresión se refiere a: a) la asistencia prestada fuera de las grandes instituciones, en pequeños centros residenciales, aunque también junto a la habitación del paciente (tipo y modalidad de realización de la prestación); b) la asistencia prestada en ámbitos locales por operadores públicos, voluntarios, operadores «informales» o un mixto de todos ellos (fuentes de ayuda y operadores sujetos participantes); c) la asistencia a nivel local, aunque el término local puede aplicarse a un distrito, a una calle, a un barrio (zona de usuarios y nivel administrativo).

12. Concretamente, mediante la responsabilización de los ciudadanos usuarios, la promoción de iniciativas de solidaridad y la construcción de una compleja red mixta de acciones para el bienestar (Weissert, 1998). 
la política pública deberá poner el acento en la responsabilidad de las personas singulares y deberá incluir, en todos los niveles (europeo, nacional, regional y local), organizaciones intermedias, de cooperación, de voluntariado y de gobierno de intereses sociales específicos. Pero, sobre todo, deberá encontrar una integración sinérgica con múltiples organismos e iniciativas que representan a las comunidades locales.

Esta tensión recorre desde el norte hasta el sur, desde el oeste hasta el este, de la Vieja Europa. Es un movimiento cultural, antes que político o económico. El empuje es hacia una política social inspirada en los valores de la solidaridad, de la cooperación, de la interdependencia, en resumen, hacia una nueva relacionalidad que se extienda desde los mundos vitales de la vida cotidiana de las familias, mediante los organismos intermedios, hacia las instituciones públicas y políticas. La estrategia es la de la coparticipación, del link entre familias y otras instituciones sociales, respetando las diversidades, que son una riqueza a preservar.

Para afrontar los nuevos desafíos, las políticas sociales deben configurarse como "sistemas reflexivos de bienestar». La reflexibidad debe entenderse como la capacidad de producir mayor solidaridad por el hecho de que se actúa de forma más adecuada y racional en los resultados de las propias acciones.

Las antiguas metas del moderno Estado de bienestar (como la lucha contra la pobreza, la redistribución de la renta y de las oportunidades de vida, etc.), y los nuevos desafíos (como la deshumanización de los servicios sociales y sanitarios, la insuficiente defensa de los intereses de los más débiles y de las minorías, el crecimiento de las llamadas "patologías de la modernidad», etc.), deben afrontarse con nuevos estilos de policy que ayuden a las políticas sociales a ser family friendly. Estos estilos se caracterizan por dos grandes factores. Por un lado, las nuevas condiciones de la sociedad, es decir, su creciente complejidad en términos de estructuras y objetivos familiares. Por otro, la extensión de las finalidades, que trasladan hacia una nueva concepción y práctica de la ciudadanía de la familia.

Perseguir políticas sociales family friendly significa formular e implementar intervenciones para el bienestar de una comunidad con tres objetivos precisos:

1. Producir solidaridad familiar antes que individualismo, es decir, bienes comunes entre familiares antes que aislamiento y anomia.

2. Conectar de forma sensata las diferentes necesidades y dimensiones de la vida familiar, tanto en el análisis como en las respuestas.

3. Focalizar las intervenciones en la familia-en-la-comunidad local, es decir, considerar la familia como sujeto social, no como terminal pasivo.

Remarquemos las líneas de la política social diseñadas:

- No implican una política "dirigista» hacia las personas y las familias, ni obligan hacia un particular «modelo etnocéntrico de familia», sino que, al 
contrario, configuran un sistema de servicios encaminado a acrecentar las capacidades de autonomía cultural y organizativa de las personas y de sus familias en la resolución de sus problemas de vida cotidiana.

- No conducen a descargar las responsabilidades públicas en las personas y las familias, al contrario, comportan un nuevo compromiso público. Si se quiere salir del círculo vicioso que traslada a la fragmentación del tejido social, a la ausencia de solidaridad, al aislamiento y la anomia, es necesario adoptar una estrategia cultural que refuerce a la familia (empowerment model) contra el predominio de una práctica que ayuda a las familias sólo cuando están en situaciones problema (déficit model).

El servicio social tiene la particular necesidad de reorientarse a la familia como sujeto de las políticas sociales. Podemos concebir la política familiar como un sistema programado de acciones que tiene cuatro palabras clave, entendidas como requisitos a satisfacer o ejes centrales. Tal esquema: a) tiene como objetivo la realización de la ciudadanía compleja de la familia; b) se avala de un conjunto de medios que actúan a partir del principio de subsidiariedad; c) se apoya en reglas asociativas de «bienestar plural», y d) legitima su acción a partir de los derechos humanos familiares, que son aquellos inherentes a la persona en cuanto que merece poder expresarlos en relaciones de solidaridad familiar.

\section{A. Realización de la ciudadanía compleja de la familia}

El escenario de la sociedad de los próximos años es el de una comunidad ("políticamente organizada») que debe realizar los derechos humanos articulando la ciudadanía «estatal» (la conferida por el Estado, en sus diferentes instituciones, centrales y periféricas) con la ciudadanía propia de la sociedad civil y de sus sujetos, es decir, la "ciudadanía societaria» (Donati, 1999). La familia y las asociaciones familiares se convierten en sujetos a los que son atribuidos nuevas titularidades de derechos según orientaciones de ciudadanía societaria, es decir, como expresión de una originalidad de derechos generados por una sociedad del bienestar en la que se manifiestan exigencias de autoorganización por parte de las formaciones sociales primarias y secundarias. La ciudadanía societaria debe contemplarse como una alternativa al estatalismo (por ejemplo, la ciudadanía del socialismo colectivista) y al liberalismo como pura afirmación de derechos individuales desligados de las responsabilidades comunes (la ciudadanía del mercado). La ciudadanía es cada vez más aquel conjunto de derechos y deberes que las personas expresan mediante sus formaciones sociales, en los diversos niveles territoriales, locales, regionales, nacionales y supranacionales, y según redes asociativas autónomas. 


\section{B. Principio operativo de subsidiariedad}

El principio de subsidiariedad afirma que no es lícito que una comunidad «política» (en sentido empírico o en sentido analítico) ${ }^{13}$ de orden superior asuma poderes y objetivos que corresponden a comunidades de orden inferior.

Existe una interpretación restrictiva y una más extensa: la primera se refiere a los objetivos mínimos de la comunidad menor, la segunda tiende a reforzar el radio de acción. Puede existir una interpretación simplemente defensiva, a favor de las comunidades más pequeñas, pero también puede darse una interpretación "promocional», en el sentido de que las comunidades mayores no sólo deben ejercer la tutela, sino que también deben acrecentar la autonomía de las más pequeñas, creando las condiciones para el desarrollo de tal autonomía y, en concreto, regulando los intercambios sociales (distributivos, redistributivos, conmutativos), de tal forma que las comunidades más pequeñas tengan recursos necesarios para realizar sus objetivos.

Traducido en palabras más simples, el principio significa:

a) Por lo que respecta al Estado, las decisiones político-administrativas deben ser tomadas lo más próximas posible a los ciudadanos.

b) Por lo que respecta a las relaciones entre el Estado y los otros actores (subsistemas) de la sociedad (llamada «sociedad civil» por no estar investida de las responsabilidades estatales), debe valer un principio operativo que valorice las recíprocas autonomías funcionales.

Este principio de valorización de la autonomía comporta una orientación (que es una forma de governance social) a la mayor sinergia posible entre los diferentes sujetos de la sociedad (institucional y civil), más que la colonización de las comunidades inferiores por parte de las superiores. La subsidiariedad debe entenderse no como intervención residual (según algunos, el Estado interviene si no existen otros actores, interpretación que puede considerarse errónea), sino como principio que permite equilibrar orgánicamente los diferentes niveles y funciones de la sociedad de manera integrada, sin indebidas sustituciones, de tal forma que se favorezca la respectiva responsabilidad de todo actor en la distinción de los objetivos.

\section{Reglas (governance) del «bienestar plural»}

Ya que el bienestar es multidimensional (económico, psicológico, social y cultural), es necesario que exista una pluralidad de sujetos que lo generen

13. «Político», en sentido empírico, es el carácter de todo sistema administrativo de relevancia estatal (estado, regiones, provincias, ayuntamientos, etc.) en cuanto que debe tomar decisiones colectivas vinculantes para la colectividad a la que se refiere. «Político», en sentido analítico, es el carácter de una formación social (por ejemplo, la familia) en aquello que alude a la función de tomar decisiones para el propio bien común que son vinculantes para todos sus miembros. 
y que estén legitimados para actuar como «sujetos públicos» (que no significa necesariamente estatales, sino reconocidos como sujetos de ciudadanía societaria).

Éste es el tema del bienestar plural ${ }^{14}$, en cuanto activado por formas «asociativas» que organizan el bienestar de forma «asociacional» ${ }^{15}$. Es la frontera que supera el bienestar social entendido como resultado (output) de cualquier compromiso entre Estado y mercado, o bien como bienestar mixto (welfare mix) (las llamadas «economías mixtas», como mezcla entre público y privado, en el sentido de Estado y mercado).

Una sociedad compleja avanzada del segundo milenio no puede gobernarse por un centro pensado como vértice de la sociedad. Ni puede dejarse a un mercado configurado por átomos espontáneos. Es precisa una regulación de la sociedad que permita a los actores en juego mayores libertades conectadas con las respectivas responsabilidades, y formas de governance social (no sólo de governance político), pero, ¿de qué manera?

Es necesario ser conscientes de que no es suficiente el compromiso entre Estado y mercado, un compromiso que ha estado vigente en todos los regímenes de bienestar tras la Segunda Guerra Mundial. El binomio Estado-mercado, que ha sido el eje de la sociedad moderna, no es suficiente y no es el apropiado para tal regulación. Es preciso hacer intervenir a otros dos subsistemas. En primer lugar, un tercer sistema, el llamado «tercer sector» o «privado social», constituido por las asociaciones, el voluntariado, la cooperación de solidaridad social, las fundaciones y otras organizaciones no de beneficio y no gubernamentales. Este «tercer polo asociacional» se presenta hoy como el más dinámico, activo y capaz de absorber la ausencia de regulación que existe en el mercado, de igual forma que evita la alineación de una sociedad burocrática por vía estatal. Y, posteriormente, hay un cuarto sistema, que podemos denominar el «subsistema de las familias», es decir, el conjunto de las familias entendidas como unidades de servicios primarios y sujetos de derechos y obligaciones propias, tanto por lo que respecta a las elecciones de bienestar (por ejemplo, adquisición de bienes y servicios), como por lo referente a la organización de los mismos servicios.

En otros términos, es necesario pensar en una sociedad organizada no sobre dos subsistemas, sino sobre cuatro - el Estado, el mercado, el tercer sector y el subsistema de las familias-, como polos simétricos, es decir, en igualdad e interrelación entre sí. En cuanto que el tercer y el cuarto sector aún son los menos reconocidos, es preciso promover soluciones apropiadas. En el caso del privado social, se trata de una solución «estatutaria», es decir, reglas universales de governance social que permitan a estos sujetos actuar como actores sociales públicamente libres y responsables, legitimados en ello por los propios

14. Que en ningún momento debe confundirse con el new labour de Toni Blair o con el modelo holandés, que buscan vías en el trade off entre más libertad de mercado y más regulación del Estado.

15. Sobre el concepto asociacional, véanse Hirst (1998, 1999) y Hirst, Bader (eds.) (2001). 
estatutos y por las funciones de «servicio público» (según la acepción anterior) que deben tener para ser considerados sujetos de servicio público y no sujetos meramente privados.

\section{Los derechos humanos familiares: la familia, de "carga social» a derecho humano}

Los derechos humanos familiares son la base de la legitimación de la ciudadanía de la familia. Muchos aún no han comprendido en qué sentido y forma el hablar de ciudadanía de la familia implique algo más que el reconocimiento de los derechos del individuo. Lo que al menos conlleva dos grandes consideraciones:

1. Primero, en cuanto que los derechos individuales no son «absolutos», sino, en la familia, tienen una estructura relacional; el derecho que cada uno tiene como persona se ejerce en relación con los derechos de los otros.

2. Segundo, si es verdad que la familia puede ejercer mediaciones positivas para las personas, entonces estas mediaciones deben ser reconocidas como un bien en sí.

Afirmar la ciudadanía de la familia quiere decir reconocer y valorizar reglas y comportamientos inspirados en criterios de solidaridad, o de plena reciprocidad, que tienen su justificación en los derechos relacionales de la persona. El concepto de ciudadanía reivindica el valor positivo de estas mediaciones, antes que la legitimación de reglas y comportamientos inspirados en criterios de indiferencia o, peor, de penalización de la mediación solidaria ejercida en la familia.

Quien no comprende o no acepta la idea de una ciudadanía de la familia generalmente lo hace por el temor de que tal reconocimiento sea, de cualquier forma, "anti-democrático». Sin embargo, es necesario insistir en que, al contrario, el concepto de ciudadanía de la familia intenta extender el sentido de la democracia sustancial, evitando que una aplicación ingenua y mecánica de democracia puramente procedimental a la familia tenga efectos perversos. Promover la ciudadanía de la familia significa, en otros términos, optar por elecciones que caminan en la dirección de una real, más completa democracia que actúa para la realización de los derechos de solidaridad, participación y autonomía de las personas como individuos-en-relación los unos con los otros.

Nueva ciudadanía de la familia significa que la familia no es ya una estructura de control, ni tampoco es considerada, de hecho o en potencia, como una realidad fragmentada. El formar parte de una familia se convierte en la titularidad para el acceso a derechos de promoción, y no sólo de tutela, de los derechos individuales, según criterios de solidaridad.

Es evidente que el problema de activar tal perspectiva no es simple, es complejo, ya que reconocer la subjetividad de la familia no sólo requiere nuevas provisiones de bienestar para un determinado tipo de estructura familiar (por 
ejemplo, las madres solteras), sino un salto cualitativo en la concepción y en la práctica de la ciudadanía, que de "estatal» se convierte en "societaria», es decir, capaz de reconocer a los sujetos - incluso supraindividuales, como la familia - portadores de instancias propias, capacidades asociativas y organizativas. En numerosos textos legales, se proclama el principio de la subjetividad familiar, pero, posteriormente, las medidas propuestas no son otra cosa que ofertas de servicios impersonales y anónimos para un particular destinatario (el niño, la mujer, el anciano), como si estos individuos no viviesen en una familia: la orientación a la familia, la elección de la familia, como sujeto en sí debe convertirse en conciencia civil.

Más allá de ciertas disposiciones económicas, sólo el reconocimiento de la plena ciudadanía de la familia, con todo lo que ello implica, podría ser un buen instrumento para evitar fenómenos de malestar, injusticias y patologías sociales. Sin tal reconocimiento, sobre las familias se transfieren, de forma perversa, implícita, indirecta, no declarada, crecientes cargas de solidaridad privada, y todo ello en detrimento de la consecución de metas de equidad social y de solidaridad universal inherentes a la ciudadanía.

Si hasta hace poco tiempo ha existido una correlación inversa entre fuerza del Estado y fuerza de la familia, esta correlación histórica ya no es válida en las sociedades postmodernas y complejas. De hecho, en la necesaria distinción funcional y relacional entre Estado y familia, en ésta última debe mediar un número creciente de relaciones para una positiva funcionalidad de la colectividad. La familia no puede ser ya una realidad segmentada de la sociedad (en el sentido de que se reproduce mediante modelos idénticos a sí mismos), ni tampoco una corporación o una pequeña sociedad cerrada en la sociedad más amplia. No puede ser ya una comunidad intermedia en el sentido organicista del término. Se convierte en una relación comunitaria de intermediación entre individuo y sociedad, que expresa una concreta esfera de derechos de ciudadanía: los derechos de quien, por el hecho de vivir en una familia, debe ser reconocido y sostenido con titulaciones adecuadas (o «derechos de acceso" a garantías y derechos sociales) en el ejercicio de las mediaciones que este hecho lleva consigo.

\section{Bibliografía}

ALDER, C. F. (1998). «Le local et le mondial: comment percevoir, analyser et assumer leurs interconexions?». Revue Internationale des Sciences Sociales, núm. 117.

ARBÓS, X. (1997). «La regulación estatal y el buen gobierno», en GINER, S.; SARASA, S. (eds.). Buen gobierno y política social. Barcelona: Ariel.

ARDIGO, A (1980). Crisi di gobernavilità e mondi vitali. Bolonia: Cappelli.

- (1997). Introduzione all'analisi sociologica del welfare state y delle sue transformacioni, Milán: Angeli.

- (1990). «Il welfare nella società postcomunista». Il Progetto, núm. 60.

BANKS, S. (1995). Ethics and Values in Social Work. Londres: Macmillan.

BARRY, N. (1990). Welfare, Londres: Open University Press.

BELL, D. (1994). Las contradicciones culturales del capitalismo. Madrid: Alianza. 
Bulmer, M. (1987). The Social Basis of Communty Care. Londres: Allend and Unwin. - (1989). The Goals of Social Policy, Londres: Unwin Hyman.

- (1998). "The Rejuvenation of Community Studies? Neighbours, Networks and Policy». The Sociological Review, vol. 33, núm. 3.

Clarke, P. B. (1996). Deep Citizenship, Londres: Pluto Press.

Colozzi, I. (1987). La riforma dello Stato Sociale. Un confronto europeo. Milán: Angeli.

CotTini, G. P. (1997). Il principio de sussidarietá: fondamenti e implicazioni di policy. Milán: Universidad Católica de Milán.

Coughlin, R. M. (1980). Ideology, Public Opinion and Welfare Policy. Berkeley: Institute for International Studies.

De LeONARDIS, O. (1996). «Il welfare mix. Privatismo e sfera publica». Stato e Mecarto, núm. 46.

Di NiCOLA, P. (1998). L’uomo no è unïsola. Le reti sociali primarie nella vita quotidiana. Milán: Angeli.

DonATI, P. (1999). La ciudadanía societaria. Granada: Universidad de Granada.

- (2002). Sociologia delle politiche familiari. Roma: Carocci.

ESPING-ANDERSEN, G. (1993). Los tres mundos del bienestar. Valencia: Ediciones Alfons el Magnanim.

FalCK, H. S. (1988). Social Work: The Membership Perspective, Nueva York: Springer.

Furniss, N.; Tilton, T. (1977). The Case for the Welfare State. From Social Segurity to Social Equiality. Londres: MacMillan.

Giner, S.; SARASA, S. (1997). «Altruismo cívico y política social», en Giner, S.; SARASA, S. (eds.). Buen gobierno y política social. Barcelona: Ariel.

GomA, R. (1996). «La reestructuración de los sistemas de bienestar en Europa. Revista Internacional de Sociología, vol. 15.

Heclo, H. (1981). «Toward a New Welfare State?», en Flora, P.; Heidenheimer, A. J. (eds.), The Development of Welfare State in Europe and America. Nueva York: Transaction Books.

Herrera Gómez, M. (2001). «Política social y ciudadanía». Revista del Ministerio de Trabajo y Asuntos sociales, núm. 30.

Herrera GÓmez, M.; Castón BOYER, P. (2003). Las políticas sociales en las sociedades complejas. Barcelona: Ariel.

HiRst, P. (1998). Social Welfare and Associative Democracy. Londres: Macmillan.

- (1999). "Associazionalismo: una strategia per la inclusion». Sociologia e Politiche Sociali, a. 2, núm.3.

Hirst, P.; BADER, V. (2001). Associative Democracy, The Real Tirad Way. Londres: Fran Cass.

Iglesias de Ussel, J (1998). Familia y cambio politico en España. Madrid: Tecnos. Karpel, M. A. (1986). Family Resources. The Hidden Partner in Family Therapy. Nueva York: Guilford.

LITWAK, E. (1985). The Complementary Roles of Informal Networks and Formal Systems. Nueva York: Guilford Press.

Llano, A. (1999). Humanismo cívico. Madrid: Ariel.

Marshall T. H. (1950). Citizenship and Social Class. Cambridge: Cambridge University Press.

- (1965). Class, citizenship and social development. Nueva York: Anchor Book.

Midgley, J. (1997). Social welfare in global context. Londres: Sage.

Montoro Romero, R. (1997). «La reforma del Estado de Bienestar: derechos, deberes e igualdad de oportunidades». Revista Española de Investigaciones Sociológicas, núm. 79. 
Morán, M. L.; Benedicto, J. (2000). Jóvenes y ciudadanos. Madrid: Ministerio de Trabajo y Asuntos Sociales.

Muñoz DE Bustillo, R. (eds.) (2000). El Estado de bienestar en el cambio de siglo. Madrid: Alianza.

OCHANDO C. C. (1999). El Estado de bienestar: Objetivos, modelos y teorias explicativas. Barcelona: Ariel.

PaYne, M. (1995). Social Work and Community Care. Londres: Macmillan.

Renaut, A. (1989). L'ère de l'individu. Contribution à una historie de la subjectivitè. París: Gallimard.

RODRíGUEz CABRERO, G. (1990). «Entre la protección social y el bienestar social», en Alabarracín, J. y otros. Reflexiones sobre política económica. Madrid: Popular. RossI, G. (1997). "Il principio de sussidiarietà: caratteristiche qualificanti ed implicazioni sulla política sociale». Politiche Sociali e Servizi, núm. 1.

SANICOLA, L. (1997). Itinerari nel servizio sociale. Nápoles: Liguori.

SARASA, S.; MORENO, L. (comps.) (1995). El Estado del bienestar en la Europa del sur. CSIC/IESA.

SARTORI, G. (1993). La democracia después del comunismo. Madrid: Alianza.

SGRITTA, G. (1990). «Cittadinanza: classi, squilibri di genere e asimmetrie generazionali». La Ricerca Sociale, núm. 46.

Smith, T. W. (1995). Public Support for Public Spending, 1973-1994. Roper Center, The Public Perspective.

TAylor-Gooby, P. (1983). «Legitimation Deficit, Public Opinion and the Welfare State». Sociology, vol. 17.

Teubner, G. (1992). Autopoietic Law: A New Approach to Law and society. Nueva York: Walter de Gruyter.

TORTELLA, G. (2000). La revolución del siglo XX. Madrid: Taurus.

Weissert, W. G. (1998). "The Past and Future of Home- and Community- based Long-term Care». The Milbank Quaterly, vol. 66, núm. 2.

Wilensky, H. L. (1985). Comparative Social Policy. Theories, Methods, Findigns. San Diego: Institute of International Studies, Universidad de California. 